\title{
Constructing Pairing-Friendly Genus 2 Curves with Split Jacobian
}

\author{
Robert Dryło \\ Institute of Mathematics, Polish Academy of Sciences, \\ Warsaw School of Economics, Poland \\ r.drylo@impan.gov.pl
}

\begin{abstract}
Genus 2 curves with simple but not absolutely simple jacobians can be used to construct pairing-based cryptosystems more efficient than for a generic genus 2 curve. We show that there is a full analogy between methods for constructing ordinary pairing-friendly elliptic curves and simple abelian varieties, which are iogenous over some extension to a product of elliptic curves. We extend the notion of complete, complete with variable discriminant, and sparse families introduced in by Freeman, Scott and Teske [11] for elliptic curves, and we generalize the Cocks-Pinch method and the Brezing-Weng method to construct families of each type. To realize abelian surfaces as jacobians we use of genus 2 curves of the form $y^{2}=x^{5}+a x^{3}+b x$ or $y^{2}=x^{6}+a x^{3}+b$, and apply the method of Freeman and Satoh [10]. As applications we find some families of abelian surfaces with recorded $\rho$-value $\rho=2$ for embedding degrees $k=3,4,6,12$, or $\rho=2.1$ for $k=27,54$. We also give variable-discriminant families with best $\rho$-values.
\end{abstract}

Keywords: Pairing-friendly hyperelliptic curves, abelian varieties, Weil numbers, CM method.

\section{Introduction}

Since pairings have been introduced to design cryptographic protocols (see, e.g., $[2,3,20$, $35]$ ), one of the main problems is to construct abelian varieties suitable for these applications. Let $A / \mathbb{F}_{q}$ be an abelian variety containing an $\mathbb{F}_{q}$-rational subgroup of prime order $r$ with the embedding degree $k=\min \left\{l: r \mid\left(q^{l}-1\right)\right\}$. To implement pairing-based cryptosystems $k$ should be suitably small so that pairings of $r$-torsion points with values in the field $\mathbb{F}_{q^{k}}$ could be efficiently computed, but the discrete logarithm problem in $\mathbb{F}_{q^{k}}$ remains intractable. Furthermore, in order the arithmetic on $A$ to be more efficient, we would like that the bit size of $r$ to be close to the size of $\# A\left(\mathbb{F}_{q}\right)$. Since $\log \left(\# A\left(\mathbb{F}_{q}\right)\right) \approx \operatorname{dim} A \log (q)$, we would like the parameter $\rho=\operatorname{dim} A \log q / \log r$ to be close to one. We can achieve $\rho \approx 1$ using supersigular abelian varieties, which in each dimension have bounded embedding degrees (e.g., $k \leq 6$ or 12 for supersingular elliptic curves or abelian surfaces (see [14,29, $31])$ ). For higher security levels we use ordinary varieties, which are unlikely to be found by a random choice and require specific constructions. In practice, we mainly use elliptic curves or jacobians of hyperelliptic curves of low genus.

Pairing-friendly elliptic curves. In general, to construct an ordinary elliptic curve $E$ with an embedding degree $k$ we first find parameters $(r, t, q)$ of $E$, where $t$ is the trace of $E, q$ is the size of the field of definition, and $r$ is the order of a subgroup with the embedding degree $k$. Then we use the Complex Multiplication (CM) method to find the equation of $E$, which requires that the $\mathrm{CM}$ discriminant $d$ of $E$ is sufficiently small, where $d$ is the squarefree part the non-negative integer $4 q-t^{2}$. Parameters $(r, t, q)$ of pairing-friendly elliptic 
curves are generated either directly, like in the Cocks-Pinch method (see [11, Theorem $4.1]$ ), or are obtained as values of suitable polynomials $(r(x), t(x), q(x))$ called parametric families. The former method is very flexible and allows one to obtain the subgroup orders $r$ and discriminants $d$ of almost arbitrary size, however with $\rho$-value only around 2 . Using parametric families we can considerably improve $\rho$-values for more restricted subgroup orders and discriminants.

Miyaji, Nakabayashi and Takano [25] were the first researchers to use parametric families to characterize elliptic curves of prime orders with embedding degrees $k=3,4,6$. Scott and Barreto [32], and Galbraith et al. [15] generalized their idea to describe elliptic curves with prescribed cofactors for $k=3,4,6$. Currently constructions of families with $\rho=1$ are yet known for $k=10$ and 12, and were discovered by Freeman [8] and Barreto-Naehrig [1], respectively. Most families used in practice are so-called complete families, and are constructed by the Brezing-Weng method [4]. We now recall the general definition and classification of families introduced by Freeman, Scott and Teske [11].

Definition 1. ([11, Definition 2.7]) Let $k$ and $d$ be positive integers such that $d$ is squarefree. We say that a triple of polynomials $(r(x), t(x), q(x))$ in $\mathbb{Q}[x]$ parametrizes a family of elliptic curves with embedding degree $k$ and discriminant $d$ if the following conditions are satisfied:

1. $q(x)=p(x)^{s}$ for some $s \geq 1$ and $p(x)$ that represents primes.

2. $r(x)$ is irreducible, non-constant, integer valued, and has positive leading coefficient.

3. $r(x)$ divides $q(x)+1-t(x)$.

4. $r(x)$ divides $\Phi_{k}(t(x)-1)$, where $\Phi_{k}(x)$ is the $k$ th cyclotomic polynomial.

5. The CM equation $4 q(x)-t(x)^{2}=d y^{2}$ has infinitely many integer solutions $(x, y)$.

Properties of the CM equation lead us to the classification of families. It is clear that we can write $4 q(x)-t(x)^{2}=f(x) g(x)^{2}$, where $f(x) \in \mathbb{Z}[x]$ is square-free and $g(x) \in \mathbb{Q}[x]$. Then condition (5) implies by Siegel's theorem that $\operatorname{deg} f(x) \leq 2$ (see [8, Proposition 2.10] or Lemma 16). We say that a family is complete if $f=d$; then the CM equation is satisfied for any $x \in \mathbb{Z}$. We say that a family is complete with variable discriminant if $\operatorname{deg} f=1$; then substituting $x \leftarrow\left(d x^{2}-b\right) / a$, where $f(x)=a x+b$, yields a complete family with discriminant $d$ if conditions (1) and (2) of Definition 1 are satisfied. A family is called sparse if $\operatorname{deg} f=2$; then the $\mathrm{CM}$ equation can be transformed to the generalized Pell equation, whose solutions grow exponentially. We note that the Brezing-Weng method [4] can be generalized to construct families of the latter two types (see [7]). These families can be used to generate elliptic curves with larger discriminant, which may be desired for larger randomness of cryptosystems.

Pairing-friendly genus 2 curves. There is also a great deal of interest in constructing pairing-friendly genus 2 curves. Freeman, Stevenhagen and Streng [12] give a general method to generate pairs $(r, \pi)$ such that $\pi$ is a Weil $q$-number corresponding by the Honda-Tate theory to a simple ordinary abelian variety with embedding degree $k$ with respect to $r$. In order to realize these varieties as jacobians, we must chose $\pi$ from a suitable CM field $K$, where Weil numbers in question are characterized by the condition

$$
\mathrm{N}_{K / \mathbb{Q}}(\pi-1) \equiv \Phi_{k}(\pi \bar{\pi}) \equiv 0 \quad(\bmod r) .
$$

If $[K: \mathbb{Q}]=2 g$, then the corresponding varieties are of dimension $g$ with $\rho$-value around $2 g^{2}$. Freeman [9] generalized this method to construct parametric families of abelian vari- 
eties. In order to obtain pairing-friendly ordinary abelian surfaces, which generically have $\rho$-value around 4 , or less than 4 for parametric families, we use genus 2 curves, whose jacobian is simple but not absolutely simple. Kawazoe and Takahashi [23] use curves of the form $y^{2}=x^{5}+a x$ and a closed formula for their order [13] (see also Kachisa [21]). Freeman and Satoh [10] give a general method to construct an elliptic curve, whose Weil restriction over some extension contains an abelian surface with a given embedding degree. To realize that surface as a jacobian, they use curves of the form $y^{2}=x^{5}+a x^{3}+b x$ or $y^{2}=x^{6}+a x^{3}+b$. Recently Guillevic and Vergnaud [17] extended their method using closed formulas for the order of these curves.

Contribution. In this paper we show that there is a full analogy between methods for constructing pairing-friendly elliptic curves and simple abelian varieties which are isogenous over some extension to a product of elliptic curves. Now we outline the main idea of our method. Let $K$ be a CM field of degree $2 g$, and suppose that we have a polynomial $\pi(x, y) \in K[x, y]$ such that $q(x, y)=\pi(x, y) \bar{\pi}(x, y) \in \mathbb{Q}[x, y]$ and the image $\pi\left(\mathbb{Z}^{2}\right)$ contains "sufficiently many" Weil numbers in $K$. Then we can use $\pi(x, y)$ to generate pairing-friendly Weil numbers analogously as in the Cooks-Pinch method. If $r$ is a prime such that the system

$$
\mathrm{N}_{K / \mathbb{Q}}(\pi(x, y)-1)=\Phi_{k}(q(x, y))=0,
$$

has solutions over $\mathbb{F}_{r}$, then we check whether $\pi(x, y)$ is a Weil number for lifts $x, y \in \mathbb{Z}$ of these solutions. Since generically solutions over $\mathbb{F}_{r}$ are of the similar size as $r$, the resulting varieties have $\rho$-value $\rho=g \log q(x, y) / \log r \approx 2 g \operatorname{deg} \pi(x, y)$. Thus to obtain $\rho$-value around $2 g$, we need suitable polynomials $\pi(x, y)$ of degree one. If $K$ contains an imaginary quadratic subfield $K_{0}=\mathbb{Q}(\sqrt{-d})$, then for any $u \in K$ such that $c=u \bar{u} \in \mathbb{Q}$, the polynomial $\pi(x, y)=u(x+y \sqrt{-d})$ satisfies $q(x, y)=\pi(x, y) \bar{\pi}(x, y)=c\left(x^{2}+d y^{2}\right) \in$ $\mathbb{Q}[x, y]$, however, if $c \neq 1$, then the image $\pi\left(\mathbb{Z}^{2}\right)$ does not contain sufficiently many primes. Therefore we will use $\pi(x, y)=\zeta_{s}(x+y \sqrt{-d})$ to generate Weil numbers in the CM field $K=\mathbb{Q}\left(\zeta_{s}, \sqrt{-d}\right)$, where $\zeta_{s}$ is an $s$ th primitive root of unity and $d>0$ is a square-free integer. We note that Weil $q$-numbers of the form $\pi=\zeta_{s} \pi_{0}$ with $\pi_{0} \in \mathbb{Q}(\sqrt{-d})$ correspond to simple abelian varieties which are isogenous over $\mathbb{F}_{q^{s}}$ to a power of an elliptic curve $E / \mathbb{F}_{q}$ with the Weil $q$-number $\pi_{0}$ (see Corollary 4 ).

To generalize the Cocks-Pinch and the Brezing-Weng methods we describe in Section 3 prime finite fields and number fields, where system (1) has solutions for $\pi(x, y)=\zeta_{s}(x+$ $y \sqrt{-d}$ ), and we give explicit formulas on solutions. In Section 4 we focus on constructing genus 2 curves, whose jacobian corresponds to Weil numbers $\pi=\zeta_{s} \pi_{0}$ in a quartic CM field $K=\mathbb{Q}\left(\zeta_{s}, \sqrt{-d}\right)$. We give an algorithm to construct curves of the form $y^{2}=x^{6}+a x^{3}+b$ and $y^{2}=x^{5}+a x^{3}+b x$, which is based on the method of Freeman and Satoh (see [10, Algorithm 5.11]). In Section 5 we generalize on abelian varieties Definition 1 and classification of families of elliptic curves. In Sections 6, 7, 8 we generalize the Brezing-Weng method to construct families of each type.

As applications we give complete families of abelian surfaces $(r(x), \pi(x))$ with variable discriminant and best $\rho$-values such that $\operatorname{deg} r(x)<25$. We note that some of these families for fixed discriminants were found in [10] and [17]. Furthermore, some complete families with variable discriminant are given in [10, Section 7], where they are obtained from complete families satisfying certain conditions, but no general method to construct such families is given. We also find some families with recorded $\rho$-value $\rho=2$ for $k=3,4,6,12$, or $\rho \approx 2.1$ for $k=27,54$ (see Examples 19, 24, 27). 


\section{Background}

In this section we gather basic facts on abelian varieties, which will be needed in the sequel (for details see $[26,37-40]$ ).

Let $A / \mathbb{F}_{q}$ be a $g$-dimensional abelian variety with $q$ th Frobenius endomorphism $\pi_{A}$, and its characteristic polynomial $f_{A}$. Then we have $f_{A}\left(\pi_{A}\right)=0$, and $\# A\left(\mathbb{F}_{q}\right)=f_{A}(1)$. Furthermore, all roots of $f_{A}$ are Weil $q$-numbers. Recall that an algebraic integer $\pi$ is called a Weil q-number if $|\alpha(\pi)|=\sqrt{q}$ for every embedding $\alpha: \mathbb{Q}(\pi) \rightarrow \mathbb{C})$. We say that $A$ is simple if it is not isogenous over $\mathbb{F}_{q}$ to a product of two positive dimensional abelian varieties. By the Honda-Tate theorem the map which associates the Frobenius endomorphism $\pi_{A}$ to a simple abelian variety $A / \mathbb{F}_{q}$ induces a one-to-one correspondence between isogeny classes of simple abelian varieties over $\mathbb{F}_{q}$ and conjugacy classes of Weil $q$-numbers. Recall also that a variety $A$ is called ordinary if it has the maximum number $p^{g}$ of all $p$-torsion points over $\overline{\mathbb{F}}_{q}$, where $p=\operatorname{char} \mathbb{F}_{q}$. We have the following.

Theorem 2. ([40]) Let $A / \mathbb{F}_{q}$ be a simple abelian variety of dimension $g$ with the endomorphism algebra $K=\operatorname{End}_{\mathbb{F}_{q}}(A) \otimes \mathbb{Q}$. Then $A$ is ordinary if and only if $K=\mathbb{Q}\left(\pi_{A}\right)$ is a CM field of degree $2 g$, and $\pi_{A}, \bar{\pi}_{A}$ are relatively prime in $\mathcal{O}_{K}$. Furthermore, if $A$ is ordinary, then $f_{A}$ is the minimal polynomial of $\pi_{A}$, and

$$
\# A\left(\mathbb{F}_{q}\right)=f_{A}(1)=\mathrm{N}_{K / \mathbb{Q}}\left(\pi_{A}-1\right) .
$$

Recall that a number field $K$ is called a $C M$ field if it is an imaginary quadratic extension of a totally real field. Then $K$ has an automorphism, denoted by a bar, which commutes with every embedding $K \rightarrow \mathbb{C}$ and the complex conjugation in $\mathbb{C}$.

In this paper we are interested in simple abelian varieties, which are not absolutely simple (i.e., split over some extension of the base field).

Proposition 3. A simple ordinary abelian variety $A / \mathbb{F}_{q}$ with a Weil q-number $\pi$ splits over $\mathbb{F}_{q^{s}}$ if and only if $\mathbb{Q}\left(\pi^{s}\right) \varsubsetneqq \mathbb{Q}(\pi)$. Then $A$ is isogenous to $B^{n}$ over $\mathbb{F}_{q^{s}}$, where $B / \mathbb{F}_{q^{s}}$ is a simple abelian variety with the Weil $q^{s}$-number $\pi^{s}$.

Proof. For the sake of completeness we give a proof (see also [18, Lemma 4]). We recall that if $f_{A, q}(x)=\prod_{i=1}^{2 g}\left(x-\pi_{i}\right)$, then $f_{A, q^{s}}(x)=\prod_{i=1}^{2 g}\left(x-\pi_{i}^{s}\right)$. Since $A$ is simple and ordinary, $f_{A, q}(x)$ is irreducible, and hence all $\pi_{i}$ are conjugated. If $\mathbb{Q}\left(\pi^{s}\right) \varsubsetneqq \mathbb{Q}(\pi)$, then $f_{A, q^{s}}$ is not the minimal polynomial of $\pi^{s}$, so $A$ splits over $\mathbb{F}_{q^{s}}$. Conversely, if $A \sim B_{1} \times \cdots \times B_{m}$ for simple abelian varieties $B_{i} / \mathbb{F}_{q^{s}}$, then $f_{A, q^{s}}=f_{B_{1}} \cdots f_{B_{m}}$. Since each $B_{i}$ is ordinary, $f_{B_{i}}$ is irreducible. Furthermore, since all the numbers $\pi_{1}^{s}, \ldots, \pi_{2 g}^{s}$ are conjugated, it follows that they are exactly roots of each $f_{B_{i}}$. Hence all $f_{B_{i}}$ are equal, and from the Honda-Tate theorem it follows that all $B_{i}$ are isogenous over $\mathbb{F}_{q^{s}}$, so $A \sim B_{1}^{n}$.

Corollary 4. Let $A / \mathbb{F}_{q}$ be an ordinary simple abelian variety with a Weil q-number $\pi$, and $E / \mathbb{F}_{q}$ be an ordinary elliptic curve with a Weil q-number $\pi_{0}$.

(i) Then $A$ is isogenous to $E^{g}$ over $\mathbb{F}_{q^{n}}$ if and only if $\pi=\zeta_{s} \pi_{0}$, where $\zeta_{s}$ is an sth primitive root from unity and $s \mid n$.

(ii) If $s$ is even and $\pi=\zeta_{s} \pi_{0}$, then $A$ is isogenous to $E^{\prime g}$ over $\mathbb{F}_{q^{s / 2}}$, where $E^{\prime}$ is the quadratic twist of $E$. 
(iii) If $\pi=\zeta_{s} \pi_{0}$, then $\mathbb{Q}(\pi)=\mathbb{Q}\left(\zeta_{s}, \sqrt{-d}\right)$, where $\pi_{0} \in \mathbb{Q}(\sqrt{-d})$ and $d$ is a positive squarefree integer.

Proof. (i) By Proposition 3 we have $A \sim E^{g}$ over $\mathbb{F}_{q^{n}}$ if and only if $\pi^{n}=\pi_{0}^{n}$. So, if $s$ is the minimal integer such that $\pi^{s}=\pi_{0}^{s}$, then $\pi=\zeta_{s} \pi_{0}$, and obviously $s \mid n$.

(ii) Since $-\pi_{0}$ is the Weil $q$-number of the quadratic twists $E^{\prime}$ of $E$, and $\pi=\zeta_{s / 2}\left(-\pi_{0}\right)$, it follows from (i) that $A \sim E^{\prime g}$ over $\mathbb{F}_{q^{s / 2}}$.

(iii) Since $E$ is ordinary, $\pi_{0}^{s}$ and $\bar{\pi}_{0}^{s}$ are relatively prime. Hence $\pi^{s}=\pi_{0}^{s}$ generates $\mathbb{Q}(\sqrt{-d})$, which implies that $\zeta_{s}, \sqrt{-d} \in \mathbb{Q}(\pi)$, so $\mathbb{Q}(\pi)=\mathbb{Q}\left(\zeta_{s}, \sqrt{-d}\right)$.

\subsection{Weil numbers of pairing-friendly varieties}

Recall that the embedding degree of an abelian variety $A / \mathbb{F}_{q}$ with respect to a prime $r \mid \# A\left(\mathbb{F}_{q}\right), r \neq \operatorname{char} \mathbb{F}_{q}$, is the minimal integer $k$ such that $r \mid\left(q^{k}-1\right)$. In other words, $q(\bmod r)$ is a $k$ th primitive root of unity, or equivalently, if $r \nmid k$, it is a root of the $k$ th cyclotomic polynomial $\Phi_{k}(x)$. By Theorem 2 we have the following.

Lemma 5. ([12, Proposition 2.1]) Let $K=\mathbb{Q}(\pi)$ be a CM field of degree $2 g$, where $\pi$ is a Weil q-number corresponding to an ordinary abelian variety $A$. Let $k$ be a positive integer and $r$ be a prime such that $r \nmid k q$. Then $A$ has the embedding degree $k$ with respect to $r$ if and only if

(1) $r \mid \Phi_{k}(q)$,

(2) $r \mid \mathrm{N}_{K / \mathbb{Q}}(\pi-1)$.

\section{The generalized Cocks-Pinch method}

Let $K=\mathbb{Q}\left(\zeta_{s}, \sqrt{-d}\right)$ be a CM field of degree $2 g$, where $\zeta_{s}$ is an sth primitive root of unity and $d>0$ is a square-free integer. To generate as in the Cooks-Pinch method pairingfriendly Weil numbers of the form $\pi=\zeta_{s} \pi_{0}$ with $\pi_{0} \in \mathbb{Q}(\sqrt{-d})$, we need to find a prime finite field $\mathbb{F}_{r}$ where the system

$$
\mathrm{N}_{K(x, y) / \mathbb{Q}(x, y)}\left(\zeta_{s}(x+y \sqrt{-d})-1\right)=\Phi_{k}\left(x^{2}+d y^{2}\right)=0,
$$

has solutions, and check whether $\pi(x, y)=\zeta_{s}(x+y \sqrt{-d})$ is a Weil number for lifts $x, y \in \mathbb{Z}$ of these solutions. We describe below such prime fields $\mathbb{F}_{r}$, and give explicit formulas on solutions. We also give an analogous result for number fields in order to further generalize the Brezing-Weng.

Lemma 6. Let $R=\mathbb{Z}$ or $\mathbb{Q}[x]$, and $r \in R$ be a prime such that the residue field $R /(r)$ contains primitive roots of unity $\zeta_{k}, \zeta_{s}$ and $\sqrt{-d}$ (if $R=\mathbb{Z}$, we assume that $r$ \2dks). If $\sqrt{-d} \notin \mathbb{Q}\left(\zeta_{s}\right)$, then solutions in $R /(r)$ of system (3) are of the form

$$
x=\frac{\zeta_{s}^{-1}+\zeta_{k} \zeta_{s}}{2}, \quad y= \pm \frac{\zeta_{s}^{-1}-\zeta_{k} \zeta_{s}}{2 \sqrt{-d}} .
$$

If $\sqrt{-d} \in \mathbb{Q}\left(\zeta_{s}\right)$, then one of these pairs is a solution of (3). 
Proof. We have

$$
\mathrm{N}_{K(x, y) / \mathbb{Q}(x, y)}\left(\zeta_{s}(x+y \sqrt{-d})-1\right)=\prod_{\sigma \in \operatorname{Aut}(K)}\left(\sigma\left(\zeta_{s}\right)(x+y \sigma(\sqrt{-d}))-1\right),
$$

and

$$
x^{2}+d y^{2}=\sigma\left(\zeta_{s}\right)(x+y \sigma(\sqrt{-d})) \sigma\left(\zeta_{s}^{-1}\right)(x-y \sigma(\sqrt{-d})) .
$$

Thus (3) has the same solutions over $\mathbb{Q}\left(\zeta_{k}, \zeta_{s}, \sqrt{-d}\right)$ as systems

$$
\begin{gathered}
\sigma\left(\zeta_{s}\right)(x+y \sigma(\sqrt{-d}))=1, \\
\sigma\left(\zeta_{s}^{-1}\right)(x-y \sigma(\sqrt{-d}))=\zeta_{k},
\end{gathered}
$$

for each $\zeta_{k}$ and $\sigma \in \operatorname{Aut}(K)$. Hence

$$
x=\frac{\sigma\left(\zeta_{s}^{-1}\right)+\zeta_{k} \sigma\left(\zeta_{s}\right)}{2}, y=\frac{\sigma\left(\zeta_{s}^{-1}\right)-\zeta_{k} \sigma\left(\zeta_{s}\right)}{2 \sigma(\sqrt{-d})} .
$$

If $\sqrt{-d} \notin \mathbb{Q}\left(\zeta_{s}\right)$, then the above solutions are of the form (4), since each automorphism of $\mathbb{Q}\left(\zeta_{s}\right)$ has two extensions on $K$. If $\sqrt{-d} \in \mathbb{Q}\left(\zeta_{s}\right)$, this solution is equal to one of pairs (4). Now let $P$ be a prime ideal over $r$ in $S=R\left[\zeta_{s}, \zeta_{k}, \sqrt{-d}\right]$, and $S_{P}$ be the localization of $S$ at $P$. It follows from the assumption that $R /(r)=S / P=S_{P} / P S_{P}$. Reducing solutions (5) $\bmod P S_{P}$ we get solutions in $R /(r)$ of the desired form, sine reduction $\bmod P$ induces an isomorphism between sth and $k$ th roots of unity in $S$ and $R /(r)$ by the following fact.

Lemma 7. Let $R=\mathbb{Z}$ or $\mathbb{Q}[x]$, and $r \in R$ be a prime such that the residue field $R /(r)$ contains sth primitive roots of unity (if $R=\mathbb{Z}$, we assume that $r \backslash \backslash$ s). If $P$ is a prime ideal in $R\left[\zeta_{s}\right]$ over $r$, then $R /(r)=R\left[\zeta_{s}\right] / P$ and reduction mod $P$ induces an isomorphism between sth roots of unity in $R\left[\zeta_{s}\right]$ and $R /(r)$.

Proof. We note that $S=R\left[\zeta_{s}\right]$ is the integral closure of $R$ in the field of fractions of $S$. This is well-known for $R=\mathbb{Z}$; if $R=\mathbb{Q}[x]$, it follows from the fact that $F[x]$ is integrally closed in $F(x)$ for any field $F$; in particular for $F=\mathbb{Q}\left(\zeta_{s}\right)$. We also note that the $s$ th cyclotomic polynomial $\Phi_{s}(x)$ is irreducible over $\mathbb{Q}(x)$, because it is irreducible over $\mathbb{Q}$ and coefficients of monic factors of polynomials in $\mathbb{Q}[x]$ are algebraic over $\mathbb{Q}$. Since $R \subset S$ is an integral extension of Dedekind domains, we have $r S=\prod_{i=1}^{n} P_{i}^{e}$, where $P_{i}$ are prime ideals in $S$. Let $r_{i} \bmod r$ for $r_{i} \in R$ be different $s$ th primitive roots of unity in $R /(r)$ for $i=1, \ldots, \varphi(s)$. Since $r_{i} \bmod r$ are roots of $\Phi_{s}(x)$, after rearranging we have $P_{i}=\left(r, \zeta_{s}-r_{i}\right)$ (see [24, Proposition I.8.25]). Thus $\zeta_{s}^{j} \equiv r_{i}^{j} \bmod P_{i}$ yields an isomorphism between $s$ th roots of unity.

From Lemma 6 we obtain the following generalization of the Cocks-Pinch algorithm.

Algorithm 8. Input: A CM field $K=\mathbb{Q}\left(\zeta_{s}, \sqrt{-d}\right)$ of degree $2 g$, and a positive integer $k$. Output: A pair $(r, \pi)$ such that $r$ is a prime and $\pi=\zeta_{s} \pi_{0}$ with $\pi_{0} \in \mathbb{Q}(\sqrt{-d})$ is a Weil $q$-number corresponding to a $g$-dimensional ordinary abelian variety $A / \mathbb{F}_{q}$ with the embedding degree $k$ with respect to $r$.

1. Choose a prime $r$ such that $\operatorname{lcm}(s, k) \mid(r-1)$ and $\sqrt{-d} \in \mathbb{F}_{r}$. 
2. Let $x=\frac{\zeta_{s}^{-1}+\zeta_{k} \zeta_{s}}{2}$ and $y=\frac{\zeta_{s}^{-1}-\zeta_{k} \zeta_{s}}{2 \sqrt{-d}}$ for all primitive roots of unity $\zeta_{s}, \zeta_{k} \in \mathbb{F}_{r}$.

3. If $\sqrt{-d} \in \mathbb{Q}\left(\zeta_{s}\right)$ and $x, y$ in the previous step do not satisfy system (3), put $y:=-y$.

4. Let $x_{1}, y_{1} \in[0, r)$ be lifts of $x, y$.

5. Let $\pi=\zeta_{s}\left(x_{1}+i r+\left(y_{1}+j r\right) \sqrt{-d}\right)$ for $i, j \in[-m, m]$, where $m$ is a small integer.

6. Return $(r, \pi)$ if $q=\pi \bar{\pi}$ is prime and $x_{1}+i r \neq 0$.

We expect that solutions of system (3) behave like random elements in $\mathbb{F}_{r}$, so we generically obtain $\rho$-value $\rho=\frac{g \log \left(\left(x_{1}+i r\right)^{2}+d\left(y_{1}+j r\right)^{2}\right)}{\log r} \approx 2 g$.

Remark. If $d \equiv 3 \bmod 4$, we obtain Weil numbers $\pi=\zeta_{s} \pi_{0}$ such that $\pi_{0}$ is in the proper suborder $\mathbb{Z}[\sqrt{-d}]$. If we want to generate Weil numbers without this restriction, we can modify the above method using $\pi(x, y)=\zeta_{s}(x+y(1+\sqrt{-d}) / 2)$.

\section{Freeman-Satoh curves}

In this section we focus on constructing genus 2 curves, whose jacobian corresponds to a given Weil number $\pi=\zeta_{s} \pi_{0}$ in a quartic CM field $K=\mathbb{Q}\left(\zeta_{s}, \sqrt{-d}\right)$, where $\pi_{0} \in$ $\mathbb{Q}(\sqrt{-d})$. Since $\varphi(s)=2$ or 4 , we have $s=3,4,6,8,12$ (the quartic CM field $\mathbb{Q}\left(\zeta_{5}\right)$ contains no imaginary quadratic subfield). We note that a simple abelian surface which is not absolutely simple, may be not isogenous to the jacobian of any curve (see [28]). Since abelian surfaces corresponding to Weil numbers in question have automorphisms of order $s$, so of order 3 or 4 , first it is natural to consider genus 2 curves which have automorphisms of order 3 or 4 . We will use the following families of curves

$$
\begin{aligned}
& y^{2}=x^{6}+a x^{3}+b, \\
& y^{2}=x^{5}+a x^{3}+b x,
\end{aligned}
$$

which have automorphisms of order 3 and 4 given by $(x, y) \mapsto\left(\zeta_{3} x, y\right)$ and $(-x, i y)$, respectively (for more details on genus 2 curves with additional automorphisms see $[6,16$, 19,33]). We will need the following result due to Freeman and Satoh [10].

Lemma 9. ([10, Propositions 4.1 and 4.2]) A curve $C$ given by (6) or (7) is isomorphic to the curve $y^{2}=x^{6}+c x^{3}+1$ or $y^{2}=x^{5}+c x^{3}+x$, respectively, where $c=a / \sqrt{b}$. Furthermore, $\operatorname{Jac}(C)$ is isogenous over some extension to $E^{2}$, where $E$ is an elliptic curve with the $j$-invariant

$$
\begin{aligned}
& j(E)=2^{8} 3^{3} \frac{(2 c-5)^{3}}{(c-2)(c+2)^{3}}, \\
& j(E)=2^{6} \frac{(3 c-10)^{3}}{(c-2)(c+2)^{2}},
\end{aligned}
$$

respectively.

We now describe a method based on [10, Algorithm 5.11]. Suppose that an abelian surface $A / \mathbb{F}_{q}$ corresponding to a Weil $q$-number $\pi=\zeta_{s} \pi_{0}$ is isogenous to the jacobian of a genus 2 curve $C$ given by (6) or (7). Then $A$ is isogenous over some extension to $E^{2}$, where $E$ is an elliptic curve with the $j$-invariant given by (8) or (9), respectively. By Corollary $4, A$ is also isogenous to $E_{0}^{2}$ over $\mathbb{F}_{q^{s}}$, where $E_{0}$ is an elliptic curve with the Weil $q$-number $\pi_{0}$. Hence $E$ and $E_{0}$ are isogenous, and so $\operatorname{End}(E)$ is an order in $K_{0}=\mathbb{Q}(\sqrt{-d})$. 
In particular, if $\operatorname{End}(E)=\mathcal{O}_{K_{0}}$ is the maximal order, then $j(E)$ is a root of the Hilbert class polynomial $H_{K_{0}}(x)$. Conversely, if $j \in \mathbb{F}_{q}$ is a root of $H_{K_{0}}(x)$, and there exists $c \in \mathbb{F}_{q}$ satisfying equations (8) or (9) with $j(E)=j$, then we determine isomorphism classes over $\mathbb{F}_{q}$ of curves $y^{2}=x^{6}+a x^{3}+b$ or $y^{2}=x^{5}+a x^{3}+b x$ with $a, b \in \mathbb{F}_{q}$ satisfying $c=a / \sqrt{b}$, and verify if jacobians of these curves correspond to $\pi$. We recall that to check with high probability if the jacobian of a curve $C$ corresponds to a Weil number $\pi$ we pick a random point $P \in \operatorname{Jac}(C)$ and check if $n P=0$, where $n=\mathrm{N}(\pi-1)$. The above procedure we give below as an algorithm. The only improvement is that we admit all twists of the above curves. The following examples show that this improvement is essential.

Example 10. Let $\pi=\zeta_{3}(3+2 \sqrt{-5})$ be a Weil $q$-number with $q=\pi \bar{\pi}=29$ and $n=$ $\mathrm{N}_{K / \mathbb{Q}}(1-\pi)=1029$. Using Algorithm 11 below we find that $\pi$ corresponds to the jacobian of the curve

$$
y^{2}=4 x^{6}+26 x^{5}+7 x^{4}+11 x^{3}+24 x^{2}+27 x+4,
$$

which is a twist of the curve $y^{2}=x^{6}+5 x^{3}+1$. However, checking all $a, b, c \in \mathbb{F}_{29}$, we find that there are no curves $y^{2}=a x^{6}+b x^{3}+c$, whose jacobian corresponds to $\pi$.

Algorithm 11. Input: A square-free positive integer $d, s=3,4$, and a Weil $q$-number $\pi=\zeta_{s} \pi_{0}$ with $\pi_{0}$ in $K_{0}=\mathbb{Q}(\sqrt{-d})$. Output: A genus 2 curve over $\mathbb{F}_{q}$, whose jacobian corresponds to $\pi$, or $\emptyset$.

1. Compute the Hilbert class polynomial $H_{K_{0}}(x)$.

2. For each root $j \in \mathbb{F}_{q}$ of $H_{K_{0}}(x)$ find solutions $c \in \overline{\mathbb{F}}_{q}$ of equations (8) or (9).

3. For each solution $c$, let $C: y^{2}=x^{6}+c x^{3}+1$ or $C: y^{2}=x^{5}+c x^{3}+x$. Remove $C$ if it is not hyperelliptic.

4. If $c \notin \mathbb{F}_{q}$ and all absolute invariants of $C$ lie in $\mathbb{F}_{q}$, determine a model $C_{1} / \mathbb{F}_{q}$ of $C$ and put $C:=C_{1}$.

5. Determine all twists of $C$ over $\mathbb{F}_{q}$.

6. For each twist $C^{\prime}$ choose a random point $P \in \operatorname{Jac}\left(C^{\prime}\right)\left(\mathbb{F}_{q}\right)$ and compute $n P$, where $n=\mathrm{N}_{K / \mathbb{Q}}(\pi-1)$.

7. Return $C^{\prime}$ if $n P=0$.

In this algorithm we need to compute the Hilbert class polynomial $H_{K_{0}}(x)$, which requires that the discriminant $d$ is sufficiently small (see [36]). We also note that if a genus 2 curve $C / \overline{\mathbb{F}}_{q}$ has a model over $\mathbb{F}_{q}$, then all its absolute invariants lie in $\mathbb{F}_{q}$. The converse property is not always true, but it does hold if $C$ has automorphisms other than the identity and the hyperelliptic involution. Then a model of $C$ over $\mathbb{F}_{q}$ can be computed using the generalization of the Mestre algorithm [30] due to Cardona and Quer [5].

Remark 12. (i) In the above algorithm it usually suffices to use curves (6) or (7) if $s=3$ or 4 , respectively. However, it may happen for the CM field $K=\mathbb{Q}\left(\zeta_{12}\right)$ that we need to use curves (6) to realize Weil numbers of the form $i \pi_{0}$ with $\pi_{0} \in \mathbb{Q}(\sqrt{-3})$ (see Example 19).

(ii) For Weil numbers in the CM field $\mathbb{Q}\left(\zeta_{8}\right)$ we can usually use curves $y^{2}=x^{5}+a x$, which have automorphisms of order $8,(x, y) \mapsto\left(\zeta_{8}^{2} x, \zeta_{8} y\right)$. Originally to construct pairingfriendly curves of this form Kawazoe and Takahashi [23] used the closed formula on their order (see [13]). 
Example 13. For $K=\mathbb{Q}\left(\zeta_{3}, \sqrt{-5}\right)$ and $k=16$, we find the following parameters of an abelian surface with $\rho=4.011$, and the corresponding genus 2 curve:

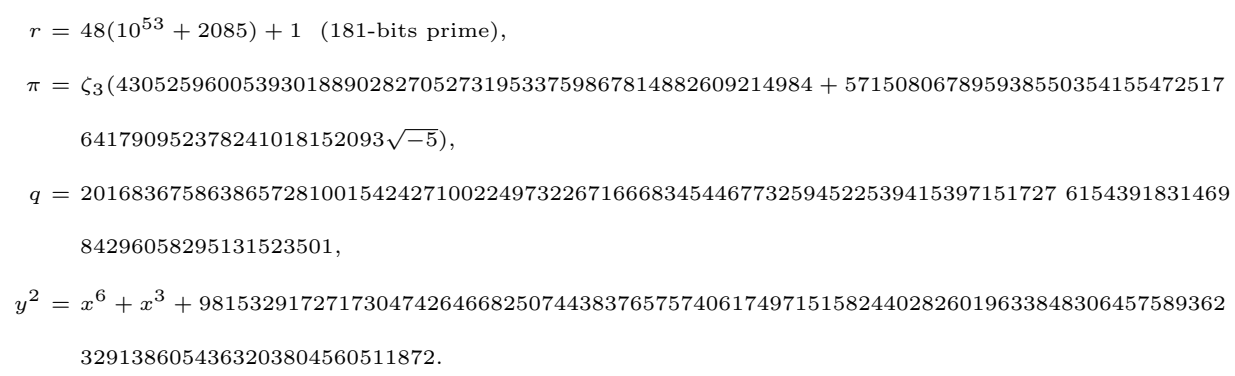

Example 14. For $K=\mathbb{Q}(i, \sqrt{-7})$ the following abelian surface has embedding degree $k=31$ and $\rho=4.016$ :

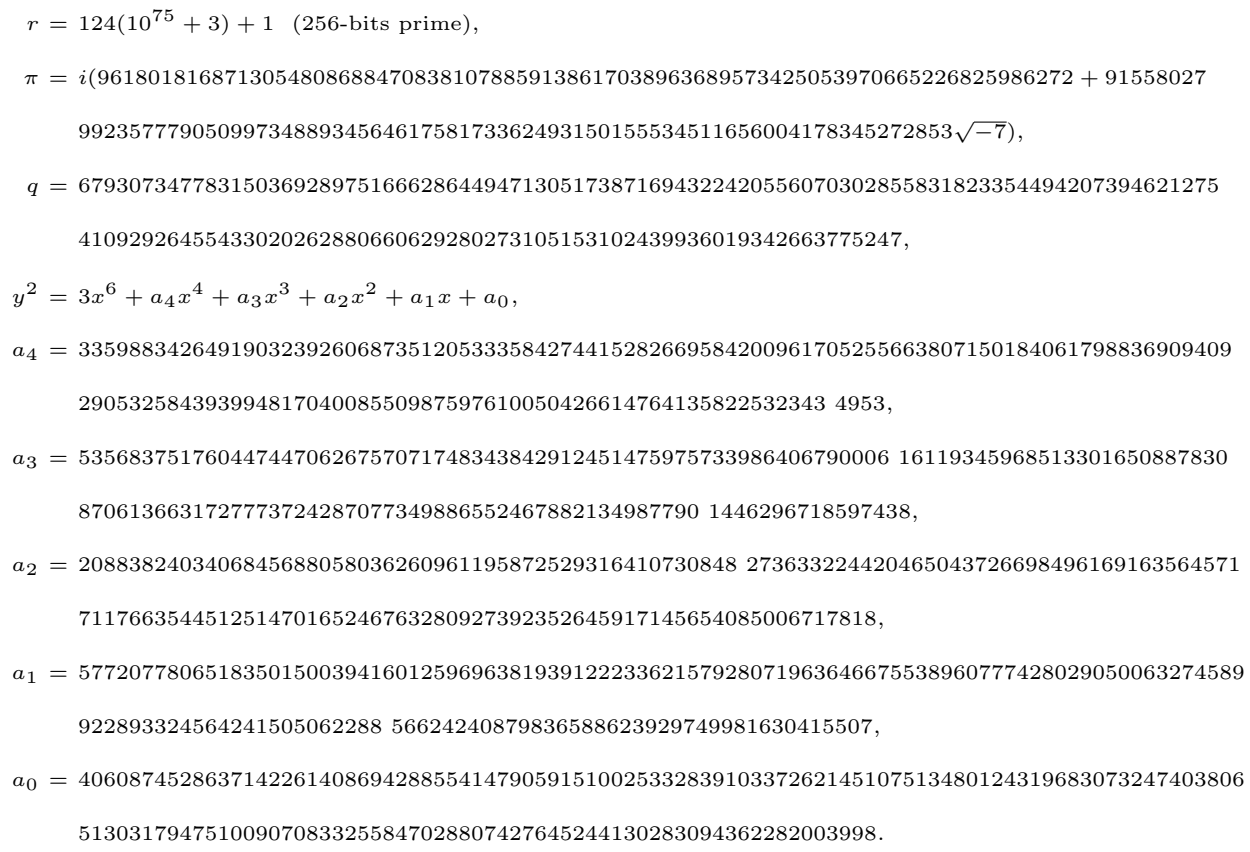

\section{$5 \quad$ Parametric Families}

Here we generalize Definition 1 and classification of families of elliptic curves on simple abelian varieties over $\mathbb{F}_{q}$, which are isogenous over some extension to a power of an elliptic curve defined over $\mathbb{F}_{q}$. Recall that by Corollary 4 Weil $q$-numbers of such abelian varieties are of the form $\pi=\zeta_{s} \pi_{0}$, where $\pi_{0}$ is a Weil $q$-number of an elliptic curve.

Definition 15. Let $K=\mathbb{Q}\left(\zeta_{s}, \sqrt{-d}\right)$ be a CM field of degree $2 g$, where $\zeta_{s}$ is an sth primitive root of unity and $d>0$ is a square-free integer. Let $r(x) \in \mathbb{Q}[x]$ and $\pi(x)=$ $\zeta_{s}\left(f_{1}(x)+f_{2}(x) \sqrt{-f(x)}\right)$, where $f_{1}(x), f_{2}(x), f(x) \in \mathbb{Q}[x]$. We say that the pair $(r(x), \pi(x))$ parametrizes a family of $g$-dimensional ordinary abelian varieties with embedding degree $k$ and discriminant $d$ if the following conditions are satisfied:

1. $q(x)=f_{1}^{2}(x)+f_{2}^{2}(x) f(x)$ is a power of a polynomial in $\mathbb{Q}[x]$ that represents primes, and $\operatorname{gcd}\left(f_{1}(x), q(x)\right)=1$. 
2. $r(x)$ is irreducible, non-constant, integer valued, and has positive leading coefficient.

3. $r(x)$ divides $N_{K_{1} / \mathbb{Q}(x)}(\pi(x)-1)$, where $K_{1}=\mathbb{Q}\left(x, \zeta_{s}, \sqrt{-f}\right)$.

4. $r(x)$ divides $\Phi_{k}(q(x))$.

5. The CM equation $f(x)=d y^{2}$ has infinitely many integer solutions $(x, y)$.

We note that the $\rho$-values $g \log q(x) / \log r(x)$ of parametrized abelian varieties tend to the $\rho$-value of the family

$$
\rho=\frac{g \operatorname{deg} q(x)}{\operatorname{deg} r(x)} .
$$

The assumption $\operatorname{gcd}\left(f_{1}(x), q(x)\right)=1$ is necessary to obtain ordinary varieties. It follows from the fact that an abelian variety with a Weil $q$-number $\pi=\zeta_{s} \pi_{0}$ is ordinary if and only if the corresponding elliptic curve with the Weil $q$-number $\pi_{0}$ is ordinary, which means that its trace $\pi_{0}+\bar{\pi}_{0}$ is relatively prime to $q$. In the examples below $q(x)$ will always represent primes, then it is sufficient that $f_{1} \neq 0$. As for elliptic curves to obtain parameters of an abelian variety with the endomorphism algebra $K=\mathbb{Q}\left(\zeta_{s}, \sqrt{-d}\right)$ we find integer solutions $\left(x_{0}, y_{0}\right)$ to the CM equation $f(x)=d y^{2}$, and check whether $\pi\left(x_{0}\right)$ is a Weil number, and $r\left(x_{0}\right)$ is prime, or almost prime. If this is the case, then $N_{K_{1} / \mathbb{Q}(x)}(\pi(x)-1)\left(x_{0}\right)$ is the order of an abelian variety corresponding to $\pi\left(x_{0}\right)$, and it is divisible by large prime factors of $r\left(x_{0}\right)$. To generalize classification of families we will need the following fact (see also $[8$, Proposition 2.10]).

Lemma 16. In Definition 15 we can assume that $f \in \mathbb{Z}[x]$ is square-free, $\operatorname{deg} f \leq 2$, and the leading coefficient of $f$ is positive.

Proof. Obviously, condition (5) in Definition 15 implies that the leading coefficient of $f$ is positive. We can write $f=g_{1} g_{2}^{2}$, where $g_{1} \in \mathbb{Z}[x]$ is square-free and $g_{2} \in \mathbb{Q}[x]$. By Siegel's theorem (see [34, Theorem IX.4.3]) the curve $d y^{2}=f(x)$ contains finitely many integer points if $f \in \mathbb{Q}[x]$ is square-free of degree $\operatorname{deg} f \geq 3$. Thus replacing $f$ by $g_{1}$ and $f_{2}$ by $f_{2} g_{2}$ we have $\operatorname{deg} f \leq 2$.

Definition 17. Let $(r(x), \pi(x))$ be a family satisfying Definition 15 with $f(x)$ as in Lemma 16 . We say that the family is

1. complete with discriminant $d$ if $f=d$,

2. complete with variable discriminant if $\operatorname{deg} f=1$,

3. sparse if $\operatorname{deg} f=2$.

The above conditions have the same interpretation as for elliptic curves, and are useful to obtain algorithms to generate families of each type, which generalize the Brezing-Weng method [4].

\section{Complete Families}

First we generalize the Brezing-Weng method [4] to construct complete families of abelian varieties. Let $K=\mathbb{Q}\left(\zeta_{s}, \sqrt{-d}\right)$ be a CM field of degree $2 g$. To construct a complete family $(r(x), \pi(x))$ with $\pi(x)=\zeta_{s}\left(f_{1}(x)+f_{2}(x) \sqrt{-d}\right)$, we need to find a number field $L=\mathbb{Q}[x] /(r(x))$ where the system

$$
\mathrm{N}_{K(x, y) / \mathbb{Q}(x, y)}\left(\zeta_{s}(x+y \sqrt{-d})-1\right)=\Phi_{k}\left(x^{2}+d y^{2}\right)=0
$$


has solutions, and take $f_{1}, f_{2} \in \mathbb{Q}[x]$ to be lifts of these solutions. Such number fields and formulas on solutions have been described in Lemma 6. Hence we have the following algorithm.

Algorithm 18. Input: A CM field $K=\mathbb{Q}\left(\zeta_{s}, \sqrt{-d}\right)$ of degree $2 g$, a positive integer $k$, and a number field $L$ containing $\zeta_{s}, \zeta_{k}, \sqrt{-d}$.

Output: A complete family $(r(x), \pi(x))$ of $g$-dimensional ordinary abelian varieties with embedding degree $k$, or $\emptyset$.

1. Find a polynomial $r(x) \in \mathbb{Q}[x]$ such that $L=\mathbb{Q}[x] /(r(x))$.

2. Let $x_{1}=\frac{\zeta_{s}^{-1}+\zeta_{k} \zeta_{s}}{2}$ and $y_{1}=\frac{\zeta_{s}^{-1}-\zeta_{k} \zeta_{s}}{2 \sqrt{-d}}$ for all $\zeta_{s}, \zeta_{k} \in L$.

3. If $\sqrt{-d} \in \mathbb{Q}\left(\zeta_{s}\right)$ and $x_{1}, y_{1}$ do not satisfy system (10), put $y_{1}=-y_{1}$.

4. Let $f_{1}, f_{2} \in \mathbb{Q}[x]$ be lifts of $x_{1}, y_{1}$ with $\operatorname{deg} f_{i}<\operatorname{deg} r, i=1,2$.

5. Let $\pi(x)=\zeta_{s}\left(f_{1}(x)+f_{2}(x) \sqrt{-d}\right)$.

6. Return $(r(x), \pi(x))$ if $f_{1} \neq 0,2 f_{1}(x) \in \mathbb{Z}$ for some $x \in \mathbb{Z}$, and $q(x)=f_{1}(x)^{2}+d f_{2}^{2}(x)$ represents primes.

We note that resulting families have $\rho$-value

$$
\rho=\frac{2 g \max \left\{\operatorname{deg} f_{1}, \operatorname{deg} f_{2}\right\}}{\operatorname{deg} r} \leq \frac{2 g(\operatorname{deg} r-1)}{\operatorname{deg} r}<2 g .
$$

In the above algorithm we can take as $L$ the cyclotomic field $L=\mathbb{Q}\left(\zeta_{s}, \zeta_{m}, \zeta_{k}\right)=\mathbb{Q}\left(\zeta_{l}\right)$, where $m$ is the smallest integer such that $\sqrt{-d} \in \mathbb{Q}\left(\zeta_{m}\right)$ and $l=\operatorname{lcm}(s, m, k)$. We note that such $m$ exists, because $\sqrt{(-1)^{\frac{p-1}{2}} p} \in \mathbb{Q}\left(\zeta_{p}\right)$ for each prime $p>2$ and $\sqrt{-2} \in \mathbb{Q}\left(\zeta_{8}\right)$ (see [27, Lemma 2.2]). Now we give a few examples; more complete families with variable discriminant will be given in Section 8 .

Example 19. Let $s=4, d=3$, and $K=\mathbb{Q}\left(\zeta_{12}\right)=\mathbb{Q}(i, \sqrt{-3})$. Let $k=12$ and $L=$ $K=\mathbb{Q}[x] /\left(r_{0}(x)\right)$, where $r_{0}(x)=x^{4}+2 x^{3}+6 x^{2}-4 x+4$ is the minimal polynomial of $\zeta_{12}-\zeta_{12}^{2}+\zeta_{12}^{3}$. Using $\pi(x, y)=i(x+y \sqrt{-3})$ we find the following family of simple ordinary abelian surfaces with embedding degree $k=12$ and $\rho=2$ :

$$
\begin{aligned}
& r(x)=\frac{1}{36}\left(x^{4}+2 x^{3}+6 x^{2}-4 x+4\right), \\
& \pi(x)=\frac{i}{12}\left(x^{2}(-\sqrt{-3}+1)-2 x(\sqrt{-3}+1)-6 \sqrt{-3}-2\right) .
\end{aligned}
$$

We note that this construction is analogous to the Barreto-Naehrig family of elliptic curves with $k=12$ and $\rho=1$ (see [1]). For example, we generate the following parameters of abelian surfaces and the corresponding genus 2 curves using Algorithm 11. 


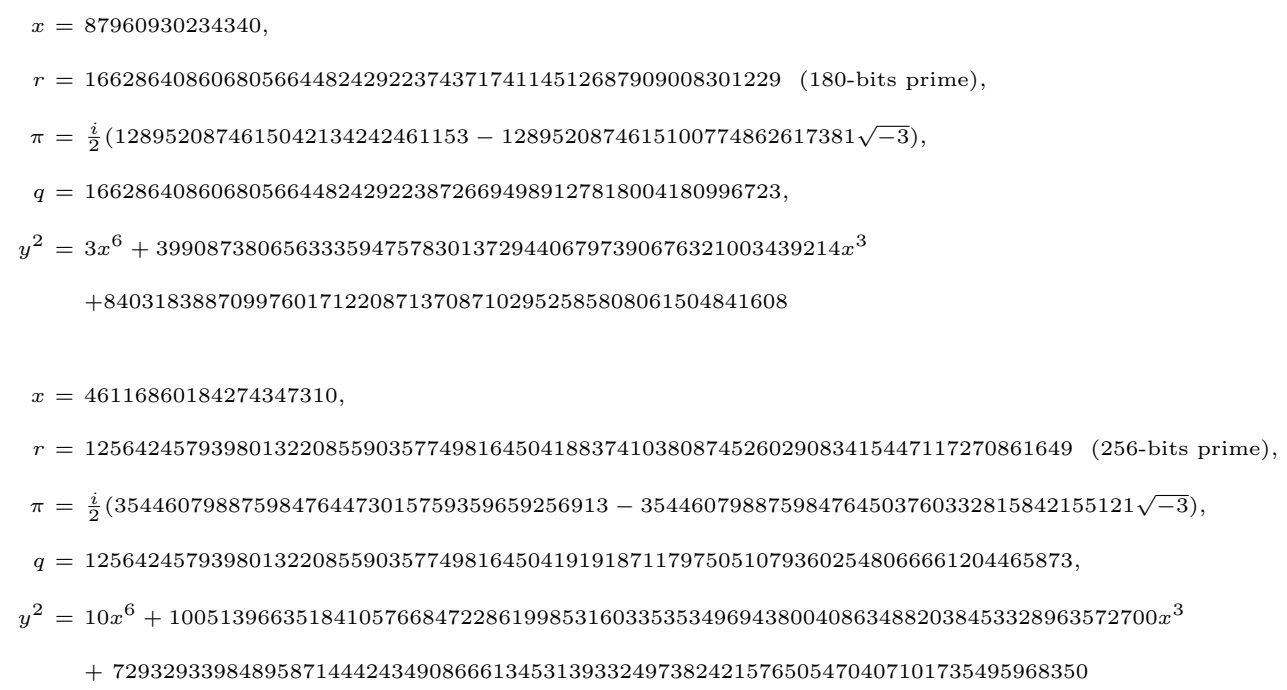

Example 20. Let $s=8, d=2$, and $K=\mathbb{Q}\left(\zeta_{8}\right)$; we have $\sqrt{-2}=\zeta_{8}^{3}+\zeta_{8}$. Using $\pi(x, y)=\zeta_{8}(x+y \sqrt{-2})$ we obtain Kawazoe-Takahashi families [23]. For example, we have the following family with $k=32$ and $\rho=3.25$ :

$$
\begin{aligned}
& r(x)=\Phi_{32}(x), \\
& \pi(x)=\frac{\zeta_{8}}{4}\left(-2 x^{13}+2 x^{12}-\sqrt{-2}\left(x^{9}+x^{8}+x+1\right)\right) .
\end{aligned}
$$

$x=1011203$

$r=r(x) / 2=597562856403016399371646603488740248049870057817560869833969493678845631715310283215375141190561$ (318-bits prime),

$\pi=-276366617178430969012422455584931203167109241914675362 \zeta_{8}^{2}-5779205224565086112079790018495549298014230975$ $89947855348929618296359476697841 \zeta_{8}+276366617178430969012422455584931203167109241914675362$,

$q=3339921302764038739820206627349052325432923549582694711656519663209495074190747019555462414145087707242326$ 37828784532408999026408517139467788305673313723369 ,

$y^{2}=x^{5}+21 x$.

Example 21. We can also give some families of 3-dimensional varieties with $\rho<6$. Constructing the corresponding genus 3 curves we leave as an open problem. The only sixtic CM fields of the form $K=\mathbb{Q}\left(\zeta_{s}, \sqrt{-d}\right)$ are the cyclotomic fields $\mathbb{Q}\left(\zeta_{7}\right)$ and $\mathbb{Q}\left(\zeta_{9}\right)$, which contain $\sqrt{-7}$ and $\sqrt{-3}$, respectively.

(i) Let $K=\mathbb{Q}\left(\zeta_{7}\right)$ and $\alpha=\sqrt{-7}=2 \zeta_{7}^{4}+2 \zeta_{7}^{2}+2 \zeta_{7}+1$. 


$$
\begin{aligned}
k=7, & \rho=4, \\
r(x) & =\Phi_{7}(x), \\
& \pi(x)=\frac{\zeta_{7}}{14}\left(-2 \alpha x^{4}+(\alpha+7) x^{3}+2 \alpha x^{2}+(\alpha+7) x-2 \alpha\right), \\
k= & 21, \quad \rho=4, \\
& r(x)=\Phi_{21}(x), \\
& \pi(x)=\frac{\zeta_{21}}{14}\left((-\alpha-7) x^{8}+(\alpha-7) x^{7}-2 \alpha x^{6}+2 \alpha x^{4}-2 \alpha x^{2}+(\alpha-7) x-\alpha-7\right) .
\end{aligned}
$$

(ii) Let $K=\mathbb{Q}\left(\zeta_{9}\right)$ and $\alpha=\sqrt{-3}=2 \zeta_{9}^{3}+1$.

$$
\begin{aligned}
& k=9, \quad \rho=4, \\
& r(x)=\Phi_{9}(x), \\
& \pi(x)=\frac{\zeta_{9}}{6}\left((-\alpha-3) x^{4}+(\alpha+3) x^{3}+(\alpha-3) x+2 \alpha\right) .
\end{aligned}
$$

\section{Sparse families}

In this section we generalize Algorithm 18 to construct sparse families in an analogous way as the Brezing-Weng method was generalized to construct such families of elliptic curves (see [7]). If $(r(x), \pi(x))$ is a family of abelian varieties with $\pi(x)=\zeta_{s}\left(f_{1}(x)+f_{2}(x) \sqrt{f(x)}\right)$, then $\left(f_{1}(x), f_{2}(x)\right) \bmod r(x)$ is a solution of the system

$$
\mathrm{N}_{K_{1} / \mathbb{Q}(x)}\left(\zeta_{s}(X+Y \sqrt{-f})-1\right)=\Phi_{k}\left(X^{2}+f Y^{2}\right)=0,
$$

where $K_{1}=\mathbb{Q}\left(x, \zeta_{s}, \sqrt{-f}\right)$. Hence to construct sparse families we should find polynomials $r(x) \in \mathbb{Q}[x]$ and $f(x) \in \mathbb{Z}[x]$, where $r(x)$ is irreducible and $f(x)$ satisfies Lemma 16, such that system (11) has solutions in the number field $L=\mathbb{Q}[x] /(r(x))$, and take $f_{1}, f_{2}$ to be lifts of these solutions. Such number fields are described in the following lemma, which generalizes Lemma 3.

Lemma 22. Let $f \in \mathbb{Z}[x]$ satisfy Lemma 16 and $\operatorname{deg} f=1,2$. Let $r(x) \in \mathbb{Q}[x]$ be irreducible such that $\zeta_{s}, \zeta_{k}, \sqrt{-\bar{f}} \in L=\mathbb{Q}[x] /(r(x))$, where a bar denotes reduction mod $r(x)$. Then system (11) has solutions in $L$ of the form

$$
X=\frac{\zeta_{s}^{-1}+\zeta_{k} \zeta_{s}}{2}, \quad Y= \pm \frac{\zeta_{s}^{-1}-\zeta_{k} \zeta_{s}}{2 \sqrt{-\bar{f}}} .
$$

Proof. As in the proof of Lemma 6 we first show that solutions in the field of fractions of $S=\mathbb{Q}\left[x, \zeta_{s}, \zeta_{k}, \sqrt{-f}\right]$ are of the above form. Then for a prime ideal $P$ in $S$ over $r$ reduction $\bmod P S_{P}$ yields the desired result by Lemma 7 .

Hence we have the following algorithm; in the next section we give a simplified version to construct complete families with variable discriminant. 
Algorithm 23. Input: A number field $L$ containing primitive roots of unity $\zeta_{s}, \zeta_{k}$. Output: A sparse family $(r(x), \pi(x))$ of $\varphi(s)$-dimensional ordinary abelian varieties with embedding degree $k$, or $\emptyset$.

1. Find $r(x) \in \mathbb{Q}[x]$ such that $L=\mathbb{Q}[x] /(r(x))$.

2. Let $f_{1} \in \mathbb{Q}[x]$ be the lift of $X=\frac{\zeta_{s}^{-1}+\zeta_{s} \zeta_{k}}{2}$ with $\operatorname{deg} f_{1}<\operatorname{deg} r$.

3. If $f_{1} \neq 0$ and $2 f_{1}(x) \in \mathbb{Z}$ for some $x \in \mathbb{Z}$, let $f(x)=a_{2} x^{2}+a_{1} x+a_{0}$ for integers $a_{0}, a_{1}, a_{2} \in[-m, m]$, where $a_{2}>0$ and $m \in \mathbb{Z}$.

4. If $f$ is square-free and $\sqrt{-\bar{f}} \in L$, let $f_{2} \in \mathbb{Q}[x]$ be the lift of $Y=\frac{\zeta_{s}^{-1}-\zeta_{s} \zeta_{k}}{2 \sqrt{-\bar{f}}}$ with $\operatorname{deg} f_{2}<\operatorname{deg} r$.

5. Let $\pi(x)=\zeta_{s}\left(f_{1}(x)+f_{2}(x) \sqrt{-f(x)}\right)$.

6. Return $(r(x), \pi(x))$ if $q(x)=f_{1}^{2}(x)+f_{2}^{2}(x) f(x)$ represents primes.

Note that the resulting families have $\rho$-value

$$
\rho=\frac{2 g \max \left\{\operatorname{deg} f_{1}, \operatorname{deg} f_{2}+1\right\}}{\operatorname{deg} r} \leq 2 g .
$$

We now show how to construct sparse families of ordinary abelian surfaces with $k=3,4,6$ and $\rho=2$. These families are analogous to constructions for elliptic curves with $k=3,4,6$ and $\rho=1$ due to Miyaji et al. [25], Scott and Barreto [32], and Galbraith et al. [15].

Example 24. Let $s=3,4$, and $K=\mathbb{Q}\left(\zeta_{s}\right)$. Let $k=3,4,6$, and $\zeta_{k} \in L=K=\mathbb{Q}[x] /(r(x))$ for $r(x) \in \mathbb{Q}[x]$. In order to construct a family $(r(x), \pi(x))$ with $\pi(x)=\zeta_{s}\left(f_{1}(x)+\right.$ $\left.f_{2}(x) \sqrt{-f(x)}\right)$ and $\rho=2$, we must find a polynomial $f(x) \in \mathbb{Z}[x]$ as in step 4 of Algorithm 23 such that $f_{2}$ is constant. Since $f_{2}$ is the lift of $Y=\left(\zeta_{s}^{-1}-\zeta_{s} \zeta_{k}\right) / 2 \sqrt{-\bar{f}}$, we must have $Y \in \mathbb{Q}$. We can assume $Y=1$, since $c^{2} f$ and $Y / c$ yield the same family for each $c \in \mathbb{Q}^{\times}$. Then for fixed $\zeta_{s}, \zeta_{k} \in L, \bar{f}$ is uniquely determined by $\bar{f}=-\left(\zeta_{s}^{-1}-\zeta_{s} \zeta_{k}\right)^{2} / 4=a \bar{x}+b$ for some $a, b \in \mathbb{Q}$. So we can take $f=a x+b+c r(x)$ for $c \in \mathbb{Q}, c>0$. As $f_{1}$ we take the lift of $X=\left(\zeta_{s}^{-1}-\zeta_{s} \zeta_{k}\right) / 2$. If $f_{1} \neq 0,2 f_{1}(x) \in \mathbb{Z}$ for some $x \in \mathbb{Z}$, and $q(x)$ represents primes, we obtain the desired family. For example, we have the following families with $\rho=2$ :

$$
\begin{aligned}
& k=3, \\
& r(x)=4 x^{2}+2 x+1, \\
& \pi(x)=\frac{\zeta_{3}}{6}\left(6 x+3+\sqrt{-\left(12 x^{2}+60 x+3\right)}\right), \\
& k=4, \\
& r(x)=4 x^{2}+1, \\
& \pi(x)=\frac{i}{2}\left(-2 x-1+\sqrt{-\left(12 x^{2}+4 x+3\right)}\right), \\
& k=6, \\
& r(x)=4 x^{2}-2 x+1, \\
& \pi(x)=\frac{\zeta_{3}}{2}\left(-2 x-1+\sqrt{-\left(12 x^{2}-4 x+3\right)}\right) .
\end{aligned}
$$


Example 25. Let $k=8, s=4$, and $L=\mathbb{Q}\left(\zeta_{8}\right)$. For $f=7 x^{2}-10 x+7$ we have $f \bmod \Phi_{8}(x)=-\left(-2 \zeta_{8}^{3}+2 \zeta_{8}^{2}-\zeta_{8}-1\right)^{2}$. We have the following family with $\rho=3$ :

$$
\begin{aligned}
& r(x)=\Phi_{8}(x), \\
& \pi(x)=\frac{i}{2}\left(-x^{2}+x+\left(2 x^{2}+3 x+2\right) \sqrt{-\left(7 x^{2}-10 x+7\right)}\right) .
\end{aligned}
$$

\section{Complete families with variable discriminant}

In this section we modify Algorithm 23 to construct complete families with variable discriminant $(r(x), \pi(x))$, where $\pi(x)=\zeta_{s}\left(f_{1}(x)+f_{2}(x) \sqrt{-f(x)}\right)$ and $f(x)=a x+b$. Substituting $x \leftarrow(x-b) / a$, we can assume that $f=x$. Then by Lemma $22, L=\mathbb{Q}[x] /(r(x))$ is a number field containing $\zeta_{s}, \zeta_{k}$, and $\sqrt{-\bar{x}}$. Let us note that a polynomial $r(x) \in \mathbb{Q}[x]$ such that $L=\mathbb{Q}[x] /(r(x))$ and $\sqrt{-\bar{x}} \in L$ can be obtained as the minimal polynomial of a primitive element $z \in L$ such that $\sqrt{-z} \in L$. Hence we have the following variant of Algorithm 23.

Algorithm 26. Input: A number field $L$ such that $\zeta_{s}, \zeta_{k} \in L$. Output: A complete family with variable discriminant $(r(x), \pi(x))$ of $\varphi(s)$-dimensional ordinary abelian varieties with embedding degree $k$, or $\emptyset$.

1. Find a primitive element $z \in L$ such that $\sqrt{-z} \in L$.

2. Let $r(x)$ be the minimal polynomial of $z$ and $L=\mathbb{Q}[x] /(r(x))$.

3. Let $X=\frac{\zeta_{s}^{-1}+\zeta_{s} \zeta_{k}}{2}$ and $Y=\frac{\zeta_{s}^{-1}-\zeta_{s} \zeta_{k}}{2 \sqrt{-\bar{x}}}$ for all $\zeta_{s}, \zeta_{k} \in L$.

4. Let $f_{1}(x), f_{2}(x) \in \mathbb{Q}[x]$ be lifts of $X, Y$ with $\operatorname{deg} f_{i}<\operatorname{deg} r, i=1,2$.

5. Let $\pi(x)=\zeta_{s}\left(f_{1}(x)+f_{2}(x) \sqrt{-x}\right)$.

6. Return $(r(x), \pi(x))$ if $f_{1} \neq 0,2 f_{1}(x) \in \mathbb{Z}$ for some $x \in \mathbb{Z}$, and $q(x)=f_{1}^{2}(x)+x f_{2}^{2}(x)$ represents primes.

The resulting families have $\rho$-value

$$
\rho=\frac{g \max \left\{2 \operatorname{deg} f_{1}, 1+2 \operatorname{deg} f_{2}\right\}}{\operatorname{deg} r} \leq \frac{g(2 \operatorname{deg} r-1)}{\operatorname{deg} r}<2 g .
$$

In the examples below we take as $L$ the cyclotomic field $L=\mathbb{Q}\left(\zeta_{s}, \zeta_{k}\right)=\mathbb{Q}\left(\zeta_{l}\right)$, where $l=\operatorname{lcm}(s, k)$. A crucial step in the above algorithm is to find a primitive element $z \in L$ such that $\sqrt{-z} \in L$, which can be chosen in the following ways:

- If $l$ is odd, then $\sqrt{\zeta_{l}}= \pm \zeta_{l}^{(l+1) / 2}$, so we can take $z=\zeta_{2 l}=-\zeta_{l}$ and $r(x)=\Phi_{2 l}(x)$. Similarly, if $l / 2$ is odd, we can take $r(x)=\Phi_{l}(x)$.

- If $4 \mid l$, then $\sqrt{ \pm \zeta_{l}} \notin \mathbb{Q}\left(\zeta_{l}\right)$, but there may exist $a \in \mathbb{Z}$ such that $\sqrt{-\zeta_{l} / a} \in \mathbb{Q}\left(\zeta_{l}\right)$. Then we can take $z=\zeta_{l} / a$ and $r(x)=\Phi_{l}(a x)$.

- As in the method of Kachisa, Schaefer, Scott [22] we can vary elements $z_{0}=a_{0}+a_{1} \zeta_{l}+$ $\cdots+a_{\varphi(l)-1} \zeta_{l}^{\varphi(l)-1}$, which have small integer coefficients in the cyclotomic basis, and use $z=-z_{0}^{2}$.

In the examples below we will also give a necessary condition on discriminant $d$ so that $q\left(d x^{2}\right)$ could represent primes. 
Example 27. (i) Let $k=27, s=3$, and $L=\mathbb{Q}\left(\zeta_{27}\right)$. We obtain the complete family with variable discriminant $d \equiv 3(\bmod 8)$ and $\rho=2.11$

$$
\begin{aligned}
& r(x)=\Phi_{54}(x), \\
& \pi(x)=\frac{\zeta_{3}}{2}\left(x^{9}-x^{5}-1-\left(x^{9}-x^{4}-1\right) \sqrt{-x}\right) .
\end{aligned}
$$

For example, we can generate the following parameters:

$$
\begin{aligned}
d & =987 \\
x & =1 \\
r & =790148551064734600930099312825768542489884551187609503 \quad(179 \text {-bits prime }) \\
\pi & =\frac{\zeta_{3}}{2}(888903004305345672187555919-888903004306281391354749065 \sqrt{-987}) \\
q & =195166692112988613822582015870901680456901569249646823659 \\
y^{2} & =x^{6}+x^{3}+151105907749622646118621216513432167109227634777454854520 \\
\rho & =2.078 \\
d & =2091 \\
x & =3 \\
r & =87647142292548622866816999275560889615442894153311051288206627370105425215463 \quad(255-\text { bits prime }) \\
\pi & =\frac{\zeta_{3}}{2}(296052600550220841104719607209577744879-888157801650662530394935347022383083571 \sqrt{-2091}) \\
q & =412379804486441270587675183690854571980192627889184083816045552664656156778750593 \\
y^{2} & =x^{6}+x^{3}+56578159329796760688848304124543683168097550241972892000909998577765239565174952 \\
\rho & =2.094
\end{aligned}
$$

(ii) Similarly, for $k=54, s=3$, and $L=\mathbb{Q}\left(\zeta_{54}\right)$, we obtain the complete family with variable discriminant $d \equiv 3(\bmod 8)$ and $\rho=2.11$

$$
\begin{aligned}
& r(x)=\Phi_{54}(x), \\
& \pi(x)=\frac{\zeta_{3}}{2}\left(x^{9}+x^{5}-1+\left(x^{9}+x^{4}-1\right) \sqrt{-x}\right) .
\end{aligned}
$$

Example 28. (i) Let $s=3, k=12$ and $L=\mathbb{Q}\left(\zeta_{12}\right)$; then $\sqrt{-\zeta_{12} / 2} \in L$. We have the following family with discriminant $d \equiv 3(\bmod 8)$ and $\rho=3.5$ :

$$
\begin{aligned}
r(x) & =\Phi_{12}(2 x), \\
f_{1}(x) & =\frac{\zeta_{3}}{2}\left(-8 x^{3}+4 x^{2}-1+\left(8 x^{3}-4 x-1\right) \sqrt{-x}\right)
\end{aligned}
$$

Example 29. Let $k=8, s=4$, and $L=\mathbb{Q}\left(\zeta_{8}\right)$. Let $r(x)$ be the minimal polynomial of $z=-\left(\zeta_{8}-1\right)^{2}$. We have the following family with discriminant $d=1,7(\bmod 8)$ and $\rho=7 / 2$ :

$$
\begin{aligned}
& r(x)=x^{4}+4 x^{3}+8 x^{2}-8 x+4 \\
& \pi(x)=\frac{i}{24}\left(-3 x^{3}-15 x^{2}-36 x+6+\left(x^{3}+5 x^{2}+16 x+2\right) \sqrt{-x}\right) .
\end{aligned}
$$


Table 1. Best $\rho$-values of complete families with variable discriminant $((r(x), \pi(x))$ such that $\operatorname{deg} r(x)<25$, which are given in the appendix.

\begin{tabular}{|c|c|c|c|c|c|c|c|}
\hline$k$ & $\rho$ & $d$ & $\operatorname{deg} r$ & $k$ & $\rho$ & $d$ & $\operatorname{deg} r$ \\
\hline 2 & 3.00 & $3(\bmod 8)$ & 2 & 22 & 2.7 & $3(\bmod 8)$ & 10 \\
3 & 3.00 & $1,3,7,9(\bmod 10)$ & 2 & 24 & 3.75 & $2,10,11,19(\bmod 24)$ & 8 \\
4 & 3.00 & $3(\bmod 4)$ & 2 & 26 & 2.25 & $3 \bmod 8$ & 24 \\
5 & 3.00 & $1(\bmod 4)$ & 8 & 27 & 2.11 & $3 \bmod 8$ & 18 \\
6 & 3.00 & any & 2 & 28 & 3.08 & $3 \bmod 8$ & 24 \\
7 & 2.50 & $3 \bmod 8$ & 12 & 30 & 2.75 & $3 \bmod 8$ & 8 \\
8 & 3.50 & $1,7(\bmod 8)$ & 4 & 33 & 2.30 & $3 \bmod 8$ & 20 \\
9 & 2.33 & $3 \bmod 8$ & 6 & 36 & 3.50 & $3 \bmod 8$ & 12 \\
10 & 3.50 & any & 8 & 39 & 2.33 & $1 \bmod 4$ & 24 \\
11 & 2.40 & $1 \bmod 4)$ & 20 & 42 & 2.83 & $3 \bmod 8$ & 12 \\
12 & 3.50 & $3 \bmod 8$ & 4 & 45 & 2.58 & $3 \bmod 8$ & 24 \\
13 & 2.25 & $3 \bmod 8$ & 24 & 54 & 2.11 & $3 \bmod 8$ & 18 \\
14 & 2.50 & $3 \bmod 8$ & 12 & 60 & 3.75 & $3 \bmod 8$ & 14 \\
15 & 2.75 & $3 \bmod 8$ & 8 & 66 & 2.30 & $3 \bmod 8$ & 20 \\
16 & 3.75 & some & 8 & 78 & 2.42 & $3 \bmod 4$ & 24 \\
18 & 2.33 & $3 \bmod 8$ & 6 & 84 & 3.75 & $3 \bmod 8$ & 24 \\
20 & 3.75 & $3 \bmod 8$ & 8 & 90 & 2.58 & $3 \bmod 8$ & 24 \\
21 & 2.66 & $1(\bmod 4)$ & 12 & & & & \\
\hline
\end{tabular}

\section{References}

1. Barreto, P.S.L.M., Naehrig, M.: Pairing-friendly elliptic curves of prime order. In Selected Areas in Cryptography - SAC 2005. LNCS, vol. 3897, pp. 319-331. Springer, Heidelberg (2006)

2. Boneh, D., Franklin, M.: Identity-based encryption from the Weil pairing. In Advances in Cryptology Crypto 2001. LNCS, vol. 2139, pp. 213-229. Springer, Berlin (2001). Full version: SIAM J. Comput. 32(3), 586-615 (2003).

3. Boneh, D., Lynn, B., Shacham, H.: Short signatures from the Weil pairing. In Advances in Cryptology Asiacrypt 2001. LNCS, vol. 2248, pp. 514-532. Springer, Berlin (2002). Full version: J. Cryptol. 17, 297-319 (2004)

4. Brezing, F., Weng, A.: Elliptic curves suitable for pairing based cryptography. Des. Codes Cryptogr. 37, 133-141 (2005)

5. Cardona, G., Quer, J.: Field of moduli and field of definition for curves of genus 2. Available at: http://arxiv.org/abs/math/0207015.

6. Cardona, G., Quer, J.: Curves of genus 2 with group of automorphisms isomorphic to $D_{8}$ or $D_{12}$. Trans. Amer. Math. Soc. 359, 2831-2849 (2007)

7. On constructing families of pairing-friendly elliptic curves with variable discriminant. INDOCRYPT2011. LNCS, vol. 7107, pp. 310-319. Springer, Berlin (2011).

8. Freeman, D.: Constructing pairing-friendly elliptic curves with embedding degree 10. In Algorithmic Number Theory Symposium - ANTS-VII. LNCS, vol. 4076, pp. 452-465. Springer, Berlin (2006).

9. Freeman, D.: A generalized Brezing-Weng algorithm for constructing pairing-friendly ordinary abelian varieties. In: Pairing-Based Cryptography - Pairing 2008. LNCS, vol. 5209, pp. 146-163. Springer, Heidelberg (2008)

10. Freeman, D., Satoh, T.: Constructing pairing-friendly hyperelliptic curves using Weil restriction. J. Number Theory 131, 959-983 (2011)

11. Freeman, D., Scott, M., Teske, E.: A taxonomy of pairing-friendly elliptic curves. J. Cryptol. 23, 224-280 (2010)

12. Freeman, D., Stevenhagen, P., Streng, M.: Abelian varieties with prescribed embedding degree. In: Algorithmic Number Theory - ANTS VIII. LNCS, vol. 5011, pp. 60-73. Springer, Heidelberg (2008)

13. Furukawa, E., Kawazoe, M., Takahashi, T.: Counting points for hyperelliptic curves of type $y^{2}=x^{5}+a x$ over finite prime fields. In Selected Areas in Cryptography - SAC 2003. LNCS, vol. 3006, pp. 26-41. Springer, Heidelberg (2004)

14. Galbraith, S.: Supersingular curves in cryptography. In ASIACRYPT 2001. LNCS, 2248, pp. 495-513. Springer, Berlin (2001). 
15. Galbraith, S., McKee, J., Valença, P.: Ordinary abelian varieties having small embedding degree. Finite Fields Appl. 13, 800-814 (2007)

16. Gaudry, P., Schost, E.: On the invariants of the quotients of the Jacobian of a curve of genus 2. In Applied Algebra, Algebraic Algorithms and Error-Correcting Codes - AAECC- 14. LNCS, vol. 2227, pp. 373-386. Springer, Heidelberg (2001)

17. Guillevic, A., Vergnaud, D.: Genus 2 Hyperelliptic Curve Families with Explicit Jacobian Order Evaluation and Pairing-Friendly Constructions. To appear in Pairing-Based Cryptography - Pairing 2012, LNCS.

18. Howe, E., Zhu, H.: On the existence of absolutely simple abelian varieties of a given dimension over an arbitrary field. J. Number Theory 92, 139-163 (2002)

19. Igusa, J.: Arithmetic Variety of Moduli for Genus Two. Ann. Math. 72, 612-649 (1960)

20. Joux A.: A one round protocol for tripartite Diffie-Hellman. In Algorithmic Number Theory Symposium - ANTS-IV. LNCS, vol. 1838, pp. 385-393. Springer, Berlin (2000). Full version: J. Cryptol. 17, 263-276 (2004)

21. Kachisa, E.: Generating More Kawazoe-Takahashi Genus 2 Pairing-Friendly Hyperelliptic Curves. In: Pairing-Based Cryptography - Pairing 2010. LNCS, vol. 6487, pp. 312-326. Springer, Heidelberg (2010).

22. Kachisa, E., Schaefer, E., Scott, M.: Constructing Brezing-Weng pairing friendly elliptic curves using elements in the cyclotomic field, in Pairing-Based Cryptography-Pairing 2008. LNCS, vol. 5209, pp. 126-135. Springer, Heidelberg (2008)

23. Kawazoe, M., Takahashi, T.: Pairing-friendly ordinary hyperelliptic curves with ordinary Jacobians of type $y^{2}=x^{5}+a x$. In: Pairing-Based Cryptography - Pairing 2008. LNCS, vol. 5209, pp. 164-177. Springer, Heidelberg (2008)

24. Lang, S.: Algebraic Number Theory. Graduate Texts in Mathematics, Vol. 110. Springer, Berlin (1994)

25. Miyaji, A., Nakabayashi, M., Takano, S.: New explicit conditions of elliptic curve traces for FRreduction. IEICE Trans. Fundam. E84-A(5), 1234-1243 (2001)

26. Milne, J.S.: Abelian varieties. In: Cornell, G., Silverman, J. (eds.) Arithmetic Geometry 103-150. Springer, New York (1986)

27. Murphy, A., Fitzpatrick, N.: Elliptic curves for pairing applications. Available at: http://eprint.iacr.org/2005/302

28. Maisner, D., Nart, E.: Abelian surfaces over finite fields as Jacobians. Experimental Mathematics 11, 321-337 (2002). With an appendix by Everett W. Howe.

29. Menezes, A., Okamoto, T., Vanstone, S.: Reducing elliptic curve logarithms to logarithms in a finite field. IEEE Trans. Inf. Theory 39, 1639-1646 (1993)

30. Mestre, J.F.: Construction de courbes de genre 2 à partir de leurs modules. In Effective methods in algebraic geometry (Castiglioncello, 1990), pages 313-334. Birkhäuser, Boston, MA (1991)

31. Rubin, K., Silverberg, A.: Using abelian varieties to improve pairing-based cryptography. J. Cryptol. 22, 330-364 (2009)

32. Scott, M., Barreto, P.S.L.M.: Generating more MNT elliptic curves. Des. Codes Cryptogr. 38, 209-217 (2006)

33. Shaska, T., Voelklein, H.: Elliptic subfields and automorphisms of genus 2 function fields. Algebra, arithmetic and geometry with applications (West Lafayette, IN, 2000), 703-723. Springer, Heidelberg (2004)

34. Silverman, J.: The Arithmetic of Elliptic Curves. Springer, Berlin (1986).

35. Sakai, R., Ohgishi, K., Kasahara, M.: Cryptosystems based on pairings. In 2000 Symposium on Cryptography and Information Security - SCIS 2000, Okinawa, Japan, 2000.

36. Sutherland, A.: Computing Hilbert class polynomials with the Chinese remainder theorem. Math. Comp. 80, 501-538 (2011)

37. Tate, J.: Classes d'isogénie des variétés abéliennes sur un corps fini. (d'aprés T. Honda.) Séminarie Bourbaki 1968/69, exposé 352. Lect. Notes in Math., vol. 179, pp. 95-110. Springer (1971)

38. Tate, J.: Endomorphisms of abelian varieties over finite fields. Inventiones Mathematicae 2 (1966)

39. Waterhouse, W.C.: Abelian varieties over finite fields. Ann. Sci. École Norm. Sup. 2, 521-560 (1969)

40. Waterhouse, W.C., Milne, J.S.: Abelian varieties over finite fields. Proc. Symp. Pure Math. 20, 53-64 (1971) 
9 Appendix: Complete families $((r(x), \pi(x))$ with variable discriminant and best $\rho$-values such that $\operatorname{deg} r(x)<25$.

$$
\begin{aligned}
& k=2, \quad \rho=3 \\
& r(x)=\Phi_{6}(x) \\
& \pi(x)=\frac{\zeta_{3}}{2}(2 x-1+x \sqrt{-x}) \\
& k=3, \quad \rho=3 \\
& r(x)=x^{2}+11 x+49 \\
& \pi(x)=\frac{\zeta_{3}}{70}(7 x+56+(x-17) \sqrt{-x}) \\
& k=4, \quad \rho=3 \\
& r(x)=x^{2}-6 x+25 \\
& \pi(x)=\frac{i}{40}(5 x+5+(x+9) \sqrt{-x}) \\
& k=5, \quad \rho=3 \\
& r(x)=\Phi_{30}(x) \\
& \pi(x)=\frac{\zeta_{3}}{2}\left(-x^{6}+x^{5}+x-1-\left(x^{3}+x^{2}\right) \sqrt{-x}\right) \\
& k=6, \quad \rho=3 \\
& r(x)=\Phi_{6}(x) \\
& \pi(x)=\frac{\zeta_{3}}{2}(x-2+(x-1) \sqrt{-x}) \\
& k=7, \quad \rho=2.5 \\
& r(x)=\Phi_{42}(x) \\
& \pi(x)=\frac{\zeta_{3}}{2}\left(x^{7}+x^{4}-1+\left(x^{7}+x^{3}-1\right) \sqrt{-x}\right) \\
& k=8, \quad \rho=3.5 \\
& r(x)=x^{4}+4 x^{3}+8 x^{2}-8 x+4 \\
& \pi(x)=\frac{i}{24}\left(-3 x^{3}-15 x^{2}-36 x+6+\left(x^{3}+5 x^{2}+16 x+2\right) \sqrt{-x}\right) \\
& k=9, \quad \rho=2.33 \\
& r(x)=\Phi_{18}(x) \\
& f_{1}(x)=\frac{\zeta_{3}}{2}\left(x^{6}-x^{3}+1+\left(x^{3}+x^{2}-1\right) \sqrt{-x}\right) \\
& k=10, \quad \rho=3.5 \\
& r(x)=\Phi_{30}(5 x) \\
& \pi(x)=\frac{\zeta_{3}}{2}\left(-78125 x^{7}-15625 x^{6}+3125 x^{5}+625 x^{4}+125 x^{3}+25 x^{2}-2\right. \\
& \left.+\left(15625 x^{6}-6250 x^{5}-1250 x^{4}-250 x^{3}+25 x^{2}\right) \sqrt{-x}\right)
\end{aligned}
$$




$$
\begin{aligned}
& k=11, \quad \rho=2.4 \\
& r(x)=\Phi_{66}(x) \\
& f_{1}(x)=\frac{\zeta_{3}}{2}\left(-x^{12}+x^{11}+x-1+\left(x^{6}+x^{5}\right) \sqrt{-x}\right) \\
& k=12, \quad \rho=3.5 \\
& r(x)=\Phi_{12}(2 x) \\
& f_{1}(x)=\frac{\zeta_{3}}{2}\left(-8 x^{3}+4 x^{2}-1+\left(8 x^{3}-4 x-1\right) \sqrt{-x}\right) \\
& k=13, \quad \rho=2.25 \\
& r(x)=\Phi_{78}(x) \\
& f_{1}(x)=\frac{\zeta_{3}}{2}\left(x^{13}-x^{7}-1+\left(x^{13}-x^{6}-1\right) \sqrt{-x}\right) \\
& k=14, \quad \rho=2.5 \\
& r(x)=\Phi_{42}(x) \\
& \pi(x)=\frac{\zeta_{3}}{2}\left(x^{7}-x^{4}-1+\left(-x^{7}+x^{3}+1\right) \sqrt{-x}\right) \\
& k=15, \quad \rho=2.75 \\
& r(x)=\Phi_{30}(x) \\
& f_{1}(x)=\frac{\zeta_{3}}{2}\left(x^{5}-x^{3}-1+\left(x^{5}-x^{2}-1\right) \sqrt{-x}\right) \\
& k=16, \quad \rho=3.75 \\
& r(x)=x^{8}+76 x^{6}+678 x^{4}+332 x^{2}+1 \\
& \pi(x)=\frac{i}{30464}\left(29 x^{7}-29 x^{6}+2173 x^{5}-2173 x^{4}+17175 x^{3}-17175 x^{2}-21009 x+5777\right. \\
& +\left(5777 x^{7}-229 x^{6}+439081 x^{5}-17389 x^{4}+3918979 x^{3}-154335 x^{2}+1935139 x\right. \\
& -71215) \sqrt{-x}) \\
& k=18, \quad \rho=2.33 \\
& r(x)=\Phi_{18}(x) \\
& \pi(x)=\frac{\zeta_{3}}{2}\left(x^{3}-x^{2}-1+\left(-x^{3}+x+1\right) \sqrt{-x}\right) \\
& k=20, \quad \rho=3.75 \\
& r(x)=\Phi_{20}(2 x) \\
& \pi(x)=\frac{i}{2}\left(-64 x^{6}+32 x^{5}+16 x^{4}-4 x^{2}+1+\left(128 x^{7}-32 x^{5}-4 x^{2}-1\right) \sqrt{-x}\right) \\
& k=21, \quad \rho=2.66 \\
& r(x)=\Phi_{42}(x) \\
& \pi(x)=\frac{\zeta_{3}}{2}\left(-x^{8}+x^{7}+x-1+\left(x^{4}+x^{3}\right) \sqrt{-x}\right)
\end{aligned}
$$




$$
\begin{aligned}
& k=22, \quad \rho=2.7 \\
& r(x)=\Phi_{22}(x) \\
& \pi(x)=\frac{\zeta_{3}}{2}\left(x^{11}-x^{8}-1+\left(-x^{13}+x^{5}+x^{2}\right) \sqrt{-x}\right) \\
& k=24, \quad \rho=3.75 \\
& r(x)=x^{8}+80 x^{6}+456 x^{4}+320 x^{2}+16 \\
& \pi(x)=\frac{\zeta_{3}}{10752}\left(-176 x^{7}+28 x^{6}-14040 x^{5}+2240 x^{4}-77088 x^{3}+12656 x^{2}-40480 x+1792\right. \\
& \left.+\left(177 x^{7}+34 x^{6}+14150 x^{5}+2704 x^{4}+79892 x^{3}+14248 x^{2}+50552 x+9024\right) \sqrt{-x}\right) . \\
& k=26, \quad \rho=2.25 \\
& r(x)=\Phi_{78}(x) \\
& \pi(x)=\frac{\zeta_{3}}{2}\left(x^{13}+x^{7}-1-\left(x^{13}+x^{6}-1\right) \sqrt{-x}\right) \\
& k=27, \quad \rho=2.11 \\
& r(x)=\Phi_{54}(x) \\
& \pi(x)=\frac{\zeta_{3}}{2}\left(x^{9}-x^{5}-1+\left(-x^{9}+x^{4}+1\right) \sqrt{-x}\right) \\
& k=28, \quad \rho=3.08 \\
& r(x)=\Phi_{84}(2 x) \\
& \pi(x)=\frac{\zeta_{3}}{2}\left(16384 x^{14}-32 x^{5}-1+\left(262144 x^{18}-131072 x^{17}+65536 x^{16}+32768 x^{15}\right.\right. \\
& \left.\left.-4096 x^{12}-1024 x^{10}+64 x^{6}-4 x^{2}-1\right) \sqrt{-x}\right) \\
& k=30, \quad \rho=2.75 \\
& r(x)=\Phi_{30}(x) \\
& \pi(x)=\frac{\zeta_{3}}{2}\left(x^{5}+x^{3}-1+\left(-x^{5}-x^{2}+1\right) \sqrt{-x}\right) \\
& k=33, \quad \rho=2.3 \\
& r(x)=\Phi_{33}(-x) \\
& f_{1}(x)=\frac{\zeta_{3}}{2}\left(x^{11}+x^{6}-1+\left(x^{11}+x^{5}-1\right) \sqrt{-x}\right) \\
& k=36, \quad \rho=3.5 \\
& r(x)=\Phi_{36}(2 x) \\
& f_{1}(x)=\frac{\zeta_{3}}{2}\left(64 x^{6}-32 x^{5}-1+\left(1024 x^{1} 0+512 x^{9}-128 x^{7}-16 x^{4}-1\right) \sqrt{-x}\right) \\
& k=39, \quad \rho=2.33 \\
& r(x)=\Phi_{78}(x) \\
& f_{1}(x)=\frac{\zeta_{3}}{2}\left(-x^{14}+x^{13}+x-1-\left(x^{7}+x^{6}\right) \sqrt{-x}\right)
\end{aligned}
$$




$$
\begin{aligned}
& k=42, \quad \rho=2.83 \\
& r(x)=\Phi_{42}(x) \\
& \pi(x)=\frac{\zeta_{3}}{2}\left(x^{7}+x^{5}-1 x+\left(x^{8}+x^{3}-x\right) \sqrt{-x}\right) \\
& k=45, \quad \rho=2.58 \\
& r(x)=\Phi_{90}(x) \\
& \pi(x)=\frac{\zeta_{3}}{2}\left(x^{15}+x^{8}-1\left(x^{15}+x^{7}-1\right) \sqrt{-x}\right) \\
& k=54, \quad \rho=2.11 \\
& r(x)=\Phi_{54}(x) \\
& \pi(x)=\frac{\zeta_{3}}{2}\left(x^{9}+x^{5}-1+\left(x^{9}+x^{4}-1\right) \sqrt{-x}\right) \\
& k=60, \quad \rho=3.75 \\
& r(x)=\Phi_{60}(2 x) \\
& \pi(x)=\frac{\zeta_{3}}{2}\left(32768 x^{15}+16384 x^{14}+4096 x^{12}-256 x^{8}-64 x^{6}-16 x^{4}+1\right. \\
& \left.+\left(4096 x^{12}+1024 x^{10}-128 x^{7}+32 x^{5}-4 x^{2}-1\right) \sqrt{-x}\right) \\
& k=66, \quad \rho=2.3 \\
& r(x)=\Phi_{66}(x) \\
& \pi(x)=\frac{\zeta_{3}}{2}\left(x^{11}-x^{6}-1+\left(-x^{11}+x^{5}+1\right) \sqrt{-x}\right) \\
& k=78, \quad \rho=2.42 \\
& r(x)=\Phi_{78}(x) \\
& \pi(x)=\frac{\zeta_{3}}{2}\left(x^{13}-x^{8}-1+\left(x^{14}-x^{6}-x\right) \sqrt{-x}\right) \\
& k=84, \quad \rho=3.75 \\
& r(x)=\Phi_{84}(2 x) \\
& \pi(x)=\frac{1}{2} \mathrm{i} g\left(16384 x^{14}+2 x-1+\left(-4194304 x^{22}-131072 x^{17}+65536 x^{16}-4096 x^{12}\right.\right. \\
& \left.\left.-2048 x^{11}-1024 x^{10}+256 x^{8}+64 x^{6}+16 x^{4}-4 x^{2}-1\right) \sqrt{-x}\right) \\
& k=90, \quad \rho=2.58 \\
& r(x)=\Phi_{90}(x) \\
& f_{1}(x)=\frac{1}{2}\left(x^{15}-x^{8}-1+\left(-x^{15}+x^{7}+1\right) \sqrt{-x}\right) .
\end{aligned}
$$

